\title{
New Unique Common Fixed Point Results for Four Mappings with $\Phi$-Contractive Type in 2-Metric Spaces*
}

\author{
Yongjie Piao, Yuanfeng Jin \\ Department of Mathematics, College of Science, Yanbian University, Yanji, China \\ Email:pyj6216@hotmail.com
}

Received May 15, 2012; revised June 15, 2012; accepted June 22, 2012

\begin{abstract}
In this paper, some new unique common fixed points for four mappings satisfying $\Phi$-contractive conditions on noncomplete 2-metric spaces are obtained, in which the mappings do not satisfy continuity and commutation. The main results generalize and improve many well-known and corresponding conclusions in the literatures.
\end{abstract}

Keywords: 2-Metric Space; Class $\Phi$; Cauchy Sequence; Coincidence Point; Unique Common Fixed Point

\section{Introduction}

There have appeared many unique common fixed point theorems of mappings with some contractive condition on 2-metric spaces. But most of them held under subsidiary conditions [1-3], for examples: commutativity of mappings or uniform boundness of mappings at some point, and so on. In [4-8], the author obtained similar results for infinite mappings with generalized contractive or quasi-contractive conditions under removing the above subsidiary conditions. These results generalized and improved many same type unique common fixed point theorems.

In this paper, by introducing a new class $\Phi$, we will discuss the existence problem of unique common fixed points for four mappings with $\Phi$-contractive type on noncomplete 2-metric spaces without any subsidiary conditions. The obtained main results in this paper further generalize and improve the corresponding results.

Here, we give some well known concepts and results.

Definition 1.1. ([4]) A 2-metric space $(X, d)$ consists of a nonempty set $X$ and a function

$d: X \times X \times X \rightarrow[0,+\infty)$ such that

1) for distant elements $x, y \in X$, there exists an $u \in X \quad$ such that $d(x, y, u) \neq 0$;

2) $d(x, y, z)=0$ if and only if at least two elements in $\{x, y, z\}$ are equal;

3) $d(x, y, z)=d(u, v, w)$, where $\{u, v, w\}$ is any permutation of $\{x, y, z\}$;

4) $d(x, y, z) \leq d(x, y, u)+d(x, u, z)+d(u, y, z)$ for all $x, y, z, u \in X$.

Definition 1.2. ([4]) A sequence $\left\{x_{n}\right\}_{n \in \mathbb{N}}$ in 2-metric

*This paper is supported by the Foundation of Education Ministry, Jilin Province, China (No: 2011[434]). space $(X, d)$ is said to be cauchy sequence, if for each $\varepsilon>0$ there exists a positive integer $N \in \mathbb{N}$ such that $d\left(x_{n}, x_{m}, a\right)<\varepsilon$ for all $a \in X$ and $n, m>N$.

Definition 1.3. ([4,5]) A sequence $\left\{x_{n}\right\}_{n \in \mathbb{N}}$ is said to be convergent to $x \in X$, if for each $a \in X$,

$$
\lim _{n \rightarrow+\infty} d\left(x_{n}, x, a\right)=0 .
$$

And write $x_{n} \rightarrow x$ and call $x$ the limit of $\left\{x_{n}\right\}_{n \in \mathbb{N}}$.

Definition 1.4. $([4,5])$ A 2-metric space $(X, d)$ is said to be complete, if every cauchy sequence in $X$ is convergent.

Definition 1.5. ([9,10]) Let $f$ and $g$ be self-maps on a set $X$. If $w=f x=g x$ for some $x \in X$, then $x$ is called a coincidence point of $f$ and $g$, and $w$ is called a point of coincidence of $f$ and $g$.

Definition 1.6. ([11]) Two mappings $f, g: X \rightarrow X$ are weakly compatible if, for every $x \in X$, holds $\operatorname{fg} x=$ $g f x$ whenever $f x=g x$.

Lemma 1.7. ([6-8]) Let $(X, d)$ be a 2-metric space and $\left\{x_{n}\right\}_{n \in \mathbb{N}}$ a sequence. If there exists $h \in[0,1)$ such that $d\left(x_{n+2}, x_{n+1}, a\right) \leq h d\left(x_{n+1}, x_{n}, a\right)$ for all $a \in X$ and $n \in \mathbb{N}$, then $d\left(x_{n}, x_{m}, x_{l}\right)=0$ for all $n, m, l \in \mathbb{N}$, and $\left\{x_{n}\right\}_{n \in \mathbb{N}}$ is a cauchy sequence.

Lemma 1.8. ([6-8]) If $(X, d)$ is a 2-metric space and sequence $\left\{x_{n}\right\}_{n \in \mathbb{N}} \rightarrow x \in X$, then

$$
\lim _{n \rightarrow+\infty} d\left(x_{n}, b, c\right)=d(x, b, c)
$$

for each $b, c \in X$.

Lemma 1.9. ([9,10]) Let $f, g: X \rightarrow X$ be weakly compatible. If $f$ and $g$ have a unique point of coincidence $w=f x=g x$, then $w$ is the unique common fixed point of $f$ and $g$. 


\section{Main Results}

Denote $\Phi$ the set of functions $\phi:\left(\mathbb{R}^{+}\right)^{5} \rightarrow \mathbb{R}^{+}$satisfying the following conditions: $\phi$ is continuous and increasing in each coordinate variable, and $\phi(t, t, t, 2 t, t)<t$ and $\phi(t, t, t, t, 2 t)<t$ for all $t>0$.

Examples Let $\phi_{1}, \phi_{2}:\left(\mathbb{R}^{+}\right)^{5} \rightarrow \mathbb{R}^{+}$be defined by

$$
\begin{gathered}
\phi_{1}\left(t_{1}, t_{2}, t_{3}, t_{4}, t_{5}\right)=a_{1} t_{1}+a_{2} t_{2}+a_{2} t_{3}+a_{4} t_{4}+a_{5} t_{5} ; \\
\phi_{2}\left(t_{1}, t_{2}, t_{3}, t_{4}, t_{5}\right)=\sum_{i=1}^{5} a_{i} \arctan t_{i},
\end{gathered}
$$

where $a_{1}, \cdots, a_{5}$ are non-negative real numbers satisfying

$$
\max \left\{a_{1}+a_{2}+a_{3}+2 a_{4}+a_{5}, a_{1}+a_{2}+a_{3}+a_{4}+2 a_{5}\right\}<1 .
$$

Then obviously, $\phi_{1}, \phi_{2} \in \Phi$.

The following theorem is the main result in this present paper.

Theorem 2.1. Let $(X, d)$ be a 2-metric space, $S, T, I$, $J: X \rightarrow X$ four single valued mappings satisfying that $S(X) \subset I(X)$ and $T(X) \subset J(X)$. Suppose that for each $x, y \in X$,

$$
\begin{gathered}
d(S x, T y, a) \leq q \phi(d(J x, I y, a), d(J x, S x, a), d(I y, T y, a), \\
d(J x, T y, a), d(I y, S x, a)), \forall a \in X
\end{gathered}
$$

where $0<q<1$ and $\phi \in \Phi$. If one of $S(X), T(X)$, $I(X)$ and $J(X)$ is complete, then $T$ and $I, S$ and $J$ have an unique point of coincidence in $X$. Further, $\{I, T\}$ and $\{S, J\}$ are weakly compatible respectively, then $S$, $T, I, J$ have an unique common fixed point in $X$.

Proof Take any element $x_{0} \in X$, then in view of the conditions $S(X) \subset I(X)$ and $T(X) \subset J(X)$, we can construct two sequences $\left\{x_{n}\right\}$ and $\left\{y_{n}\right\}$ as follows:

$$
y_{2 n}=S x_{2 n}=I x_{2 n+1}, y_{2 n+1}=T x_{2 n+1}=J x_{2 n+2}, n=0,1, \cdots .
$$

For any $n=0,1, \cdots$,

$$
\begin{aligned}
& d\left(y_{2 n}, y_{2 n+1}, a\right)=d\left(S x_{2 n}, T x_{2 n+1}, a\right) \\
& \leq q \phi\left(d\left(J x_{2 n}, I x_{2 n+1}, a\right), d\left(J x_{2 n}, S x_{2 n}, a\right),\right. \\
& \left.\quad d\left(I x_{2 n+1}, T x_{2 n+1}, a\right), d\left(J x_{2 n}, T x_{2 n+1}, a\right), d\left(I x_{2 n+1}, S x_{2 n}, a\right)\right) \\
& =q \phi\left(d\left(y_{2 n-1}, y_{2 n}, a\right), d\left(y_{2 n-1}, y_{2 n}, a\right), d\left(y_{2 n}, y_{2 n+1}, a\right),\right. \\
& \left.\quad d\left(y_{2 n-1}, y_{2 n+1}, a\right), d\left(y_{2 n}, y_{2 n}, a\right)\right) \\
& \leq q \phi\left(d\left(y_{2 n-1}, y_{2 n}, a\right), d\left(y_{2 n-1}, y_{2 n}, a\right), d\left(y_{2 n}, y_{2 n+1}, a\right),\right. \\
& \quad\left[d\left(y_{2 n-1}, y_{2 n}, a\right)+d\left(y_{2 n+1}, y_{2 n}, a\right)\right. \\
& \left.\left.\quad+d\left(y_{2 n-1}, y_{2 n}, y_{2 n+1}\right)\right], 0\right) .
\end{aligned}
$$

If $d\left(y_{2 n-1}, y_{2 n}, y_{2 n+1}\right)>0$, then by (1) and $\Phi$, we have that

$$
\begin{aligned}
d & \left(y_{2 n-1}, y_{2 n}, y_{2 n+1}\right)=d\left(S x_{2 n}, T x_{2 n+1}, y_{2 n-1}\right) \\
\leq & q \phi\left(d\left(J x_{2 n}, I x_{2 n+1}, y_{2 n-1}\right), d\left(J x_{2 n}, S x_{2 n}, y_{2 n-1}\right),\right. \\
& d\left(I x_{2 n+1}, T x_{2 n+1}, y_{2 n-1}\right), d\left(J x_{2 n}, T x_{2 n+1}, y_{2 n-1}\right) \\
& \left.d\left(I x_{2 n+1}, S x_{2 n}, y_{2 n-1}\right)\right) \\
= & q \phi\left(0,0, d\left(y_{2 n-1}, y_{2 n}, y_{2 n+1}\right), 0,0\right) \\
< & q d\left(y_{2 n-1}, y_{2 n}, y_{2 n+1}\right)
\end{aligned}
$$

which is a contradiction since $0<q<1$, hence $d\left(y_{2 n-1}, y_{2 n}, y_{2 n+1}\right)=0$. And therefore, (3) becomes that

$$
\begin{aligned}
& d\left(y_{2 n}, y_{2 n+1}, a\right)=d\left(S x_{2 n}, T x_{2 n+1}, a\right) \\
& \leq q \phi\left(d\left(y_{2 n-1}, y_{2 n}, a\right), d\left(y_{2 n-1}, y_{2 n}, a\right), d\left(y_{2 n}, y_{2 n+1}, a\right),\right. \\
& \left.\quad\left[d\left(y_{2 n-1}, y_{2 n}, a\right)+d\left(y_{2 n+1}, y_{2 n}, a\right)\right], 0\right) .
\end{aligned}
$$

If there exists an $a \in X$ such that $d\left(y_{2 n-1}, y_{2 n}, a\right)<d\left(y_{2 n}, y_{2 n+1}, a\right)$, then (5) becomes

$$
\begin{aligned}
& d\left(y_{2 n}, y_{2 n+1}, a\right)=d\left(S x_{2 n}, T x_{2 n+1}, a\right) \\
& \leq q \phi\left(d\left(y_{2 n}, y_{2 n+1}, a\right), d\left(y_{2 n}, y_{2 n+1}, a\right), d\left(y_{2 n}, y_{2 n+1}, a\right),\right. \\
& \left.\quad 2 d\left(y_{2 n+1}, y_{2 n}, a\right), d\left(y_{2 n}, y_{2 n+1}, a\right)\right) \\
& <q d\left(y_{2 n}, y_{2 n+1}, a\right)
\end{aligned}
$$

which is a contradiction since $0<q<1$, hence we have that $d\left(y_{2 n-1}, y_{2 n}, a\right) \geq d\left(y x_{2 n}, y_{2 n+1}, a\right)$ for all $a \in X$. So by (5) and $\Phi$, we obtain that

$$
d\left(y_{2 n}, y_{2 n+1}, a\right) \leq q d\left(y_{2 n-1}, y_{2 n}, a\right) .
$$

Similarly, we can prove that

$$
d\left(y_{2 n+1}, y_{2 n+2}, a\right) \leq q d\left(y_{2 n}, y_{2 n+1}, a\right) .
$$

Hence we have that

$$
d\left(y_{n+1}, y_{n+2}, a\right) \leq q d\left(y_{n}, y_{n+1}, a\right), \forall n=0,1, \cdots .
$$

So $\left\{y_{n}\right\}$ is a Cauchy sequence by Lemma 1.7.

Suppose that $I(X)$ is complete, then there exists $u \in I(X)$ and $v \in X$ such that

$y_{2 n}=S x_{2 n}=I x_{2 n+1} \rightarrow u=I v$. (If $S(X)$ is complete, there exists $u \in S(X) \subset I(X)$, then the conclusions remains the same.)

Since

$$
\begin{aligned}
d\left(y_{2 n+1}, u, a\right) \leq & d\left(y_{2 n+1}, y_{2 n}, a\right)+d\left(y_{2 n}, u, a\right) \\
& +d\left(y_{2 n}, y_{2 n+1}, u\right)
\end{aligned}
$$

and $\left\{y_{n}\right\}$ is Cauchy sequence and $\left\{y_{2 n}\right\} \rightarrow u$, we obtain that $\left\{y_{2 n+1}\right\} \rightarrow u$.

For any $a \in X$, 


$$
\begin{aligned}
d & (u, T v, a) \\
\leq & d\left(y_{2 n}, T v, a\right)+d\left(u, y_{2 n}, a\right)+d\left(u, y_{2 n}, T v\right) \\
= & d\left(S x_{2 n}, T v, a\right)+d\left(u, y_{2 n}, a\right)+d\left(u, y_{2 n}, T v\right) \\
\leq & q \phi\left(d\left(J x_{2 n}, I v, a\right), d\left(J x_{2 n}, S x_{2 n}, a\right), d(I v, T v, a),\right. \\
& \left.d\left(J_{2 n}, T v, a\right), d\left(I v, S x_{2 n}, a\right)\right) \\
& +d\left(u, y_{2 n}, a\right)+d\left(u, y_{2 n}, T v\right) \\
= & q \phi\left(d\left(y_{2 n-1}, u, a\right), d\left(y_{2 n-1}, y_{2 n}, a\right), d(u, T v, a),\right. \\
& \left.d\left(y_{2 n-1}, T v, a\right), d\left(u, y_{2 n}, a\right)\right) \\
& +d\left(u, y_{2 n}, a\right)+d\left(u, y_{2 n}, T v\right) .
\end{aligned}
$$

Let $n \rightarrow \infty$, then the above becomes

$$
d(u, T v, a) \leq q \phi(0,0, d(u, T v, a), d(u, T v, a), 0) .
$$
that

If $d(u, T v, a)>0$ for some $a \in X$, then we obtain

$$
d(u, T v, a)<q d(u, T v, a),
$$

which is a contradiction since $0<q<1$. Hence $d(u, T v, a)=0$ for all $x \in X$, so $T v=u=I v$, i.e., $u$ is a point of coincidence of $T$ and $I$, and $v$ is a coincidence point of $T$ and $I$.

On the other hand, since $u=T v \in T(X) \subset J(X)$, there exists $w \in X$ such that $u=J w$. By (1), for any $a \in X$,

$$
\begin{aligned}
d & (S w, u, a) \\
\leq & d\left(S w, y_{2 n+1}, a\right)+d\left(y_{2 n+1}, u, a\right)+d\left(y_{2 n+1}, u, S w\right) \\
= & d\left(S w, T x_{2 n+1}, a\right)+d\left(y_{2 n+1}, u, a\right)+d\left(y_{2 n+1}, u, S w\right) \\
\leq & q \phi\left(d\left(J w, I x_{2 n+1}, a\right), d(J w, S w, a), d\left(I x_{2 n+1}, T x_{2 n+1}, a\right),\right. \\
& \left.d\left(J w, T x_{2 n+1}, a\right), d\left(I x_{2 n+1}, S w, a\right)\right) \\
& +d\left(y_{2 n+1}, u, a\right)+d\left(y_{2 n+1}, u, S w\right) \\
= & q \phi\left(d\left(u, y_{2 n}, a\right), d(u, S w, a), d\left(y_{2 n}, y_{2 n+1}, a\right),\right. \\
& \left.d\left(u, y_{2 n+1}, a\right), d\left(y_{2 n}, S w, a\right)\right) \\
& +d\left(y_{2 n+1}, u, a\right)+d\left(y_{2 n+1}, u, S w\right) .
\end{aligned}
$$

Let $n \rightarrow \infty$, then we obtain that

$$
d(S w, u, a) \leq q \phi(0, d(S w, u, a), 0,0, d(S w, u, a)) .
$$

If $d(S w, u, a)>0$ for some $a \in X$, then the above becomes that

$$
d(S w, u, a)<q d(S w, u, a),
$$

which is a contradiction since $0<q<1$, so $d(S w, u, a)=$ for all $a \in X$. Hence $S w=u=J w$, i.e., $u$ is a point of coincidence of $S$ and $J$, and $w$ is a coincidence point of $S$ and $J$.

If $z=S X=J X$ is another point of coincidence of $S$ and $J$, then there exists $a \in X$ such that $d(z, u, a)>0$, and we have that

$$
\begin{aligned}
& d(z, u, a)=d(S x, T v, a) \\
& \leq q \phi(d(J x, I v, a), d(J x, S x, a), d(I v, T v, a), \\
& d(J x, T v, a), d(\operatorname{Iv}, S x, a)) \\
& =q \phi(d(z, u, a), d(z, z, a), d(u, u, a), \\
& \quad d(z, u, a), d(u, z, a)) \\
& <q d(z, u, a),
\end{aligned}
$$

which is a contradiction. So $d(z, u, a)=0$ for all $a \in X$, hence $z=u$, i.e., $u$ is the unique point of coincidence of $S$ and $J$. Similarly, we can prove that $u$ is also unique point of coincidence of $T$ and $I$.

By Lemma 1.9, $u$ is the unique common fixed point $\{S, J\}$ and $\{T, I\}$ respectively, hence $u$ is the unique common fixed point of $S, T, I, J$.

If $J(X)$ or $T(X)$ is complete, then we can also use similar method to prove the same conclusion. We omit the part.

Here, we give only one of particular forms of theorem 2.1, which itself also generalize and improve many known results.

Theorem 2.2. Let $(X, d)$ be a 2-metric space, $S, T, I$, $J: X \rightarrow X$ four single valued mappings satisfying that $S(X) \subset I(X)$ and $T(X) \subset J(X)$. Suppose that for each $x, y \in X$,

$$
\begin{aligned}
& d(S x, T y, a) \\
& \leq a_{1} d(J x, I y, a)+a_{2} d(J x, S x, a)+a_{3} d(I y, T y, a) \\
& \left.+a_{4} d(J x, T y, a)+a_{5} d(I y, S x, a)\right), \quad \forall a \in X,
\end{aligned}
$$

where $a_{1}, \cdots, a_{5}$ are non-negative real numbers satisfying

$$
\max \left\{a_{1}+a_{2}+a_{3}+2 a_{4}+a_{5}, a_{1}+a_{2}+a_{3}+a_{4}+2 a_{5}\right\}<1 .
$$

If one of $S(X), T(X), I(X)$ and $J(X)$ is complete, then $T$ and $I, S$ and $J$ have an unique point of coincidence in $X$. Further, $\{I, T\}$ and $\{S, J\}$ are weakly compatible respectively, then $S, T, I, J$ have an unique common fixed point in $X$.

Proof Take $0<q<1$ satisfying

$$
\frac{\max \left\{a_{1}+a_{2}+a_{3}+2 a_{4}+a_{5}, a_{1}+a_{2}+a_{3}+a_{4}+2 a_{5}\right\}}{q}<1
$$

and let

$$
\phi\left(t_{1}, t_{2}, t_{3}, t_{4}, t_{5}\right)=\frac{a_{1}}{q} t_{1}+\frac{a_{2}}{q} t_{2}+\frac{a_{3}}{q} t_{3}+\frac{a_{4}}{q} t_{5}+\frac{a_{5}}{q} t_{5} .
$$

Then obviously, $\phi \in \Phi$, hence $q$ and $\phi$ satisfy all conditions of Theorem 2.1, so the conclusion follows from theorem 2.1

Using Theorem 2.1, we give the following contractive 
or quasi-contractive versions of Theorem 2.1 for two mappings.

Corollary 2.3 Let $(X, d)$ be a 2-metric space, $S, T: X \rightarrow X$ two single valued mappings satisfying that for each $x, y \in X$,

$$
\begin{gathered}
d(S x, T y, a) \leq q \phi(d(x, y, a), d(x, S x, a), d(y, T y, a), \\
d(x, T y, a), d(y, S x, a)), \forall a \in X,
\end{gathered}
$$

where $0<q<1$ and $\phi \in \Phi$. If one of $S(X)$ and $T(X)$ is complete, then $T$ and $S$ have an unique common fixed point in $X$.

Proof Let $I=J=1_{X}$, then by Theorem 2.1, there exist $u \in X$ such that $u$ is the unique point of coincidence of $S$ and $J$. But obviously $S$ and $J$ are weakly compatible, so $u$ is the unique fixed point of $S$ by Lemma 1.9. Similarly, $u$ is also unique fixed point of $T$, hence $u$ is the unique common fixed point of $S$ and $T$.

Corollary 2.4 Let $(X, d)$ be a complete 2-metric space, $I, J: X \rightarrow X$ two single valued surjective mappings satisfying for each $x, y \in X$,

$$
\begin{gathered}
d(x, y, a) \leq q \phi(d(J x, I y, a), d(J x, x, a), d(I y, y, a), \\
d(J x, y, a), d(I y, x, a)), \forall a \in X,
\end{gathered}
$$

where $0<q<1$ and $\phi \in \Phi$, then $I$ and $J$ have an unique common fixed point in $X$.

Proof Let $S=T=1_{X}$, then by Theorem 2.1, there exist $u \in X$ such that $u$ is the unique point of coincidence of $S$ and $J$. But obviously $S$ and $J$ are weakly compatible, so $u$ is the unique fixed point of $J$ by Lemma 1.9. Similarly, $u$ is also unique fixed point of $I$, hence $u$ is the unique common fixed point of $I$ and $J$.

\section{REFERENCES}

[1] Y. J. Piao, G. Z. Jin and B. J. Zhang, "A Family of Selfmaps Having an Unique Common Fixed Point in 2-Metric Spaces," Journal of Yanbian University (Science Edition), Vol. 28, No. 1, 2002, pp. 1-5.

[2] H. S. Yang and D. S. Xiong, "A Common Fixed Point Theorem on p-Metric Spaces," Journal of Yunnan Nor- mal University (Science Edition), Vol. 21, No. 1, 2001, pp. 9-12.

[3] S. L. Singh,, "Some Contractive Type Principles on 2Metric Spaces and Applications," Mathematics Seminar Notes (Kobe University), Vol. 7, No. 1, 1979, pp. 1-11.

[4] Y. J. Piao and Y. F. Jin, "Unique Common Fixed Point Theorem for a Family of Contractive Type Non-Commuting Selfmaps in 2-Metric Spaces," Journal of Yanbian University (Science Edition), Vol. 32, No. 1, 2006, pp. 1-3 (in Chinese).

[5] Y. J. Piao, "A Family of Quasi-Contractive Type NonCommutative Self-Maps Having an Unique Common Fixed Point in 2-Metric Spaces," Journal of Heilongjiang University (Science Edition), Vol. 23, No. 5, 2006, pp. 655-657 (in Chinese).

[6] Y. J. Piao, "Unique Common Fixed Point for a Family of Self-Maps with Same Type Contractive Condition in 2Metric Spaces," Analysis in Theory and Applications, Vol. 24, No. 4, 2008, pp. 316-320. doi:10.1007/s10496-008-0316-9

[7] Y. J. Piao, "Unique Common Fixed Point for a Family of Self-Maps with Same Quasi-Contractive Type Condition in 2-Metric Space," Journal of Nanjing University (Mathematical Biquarterly), Vol. 27, No. 1, 2010, pp. $82-87$ (in Chinese).

[8] Y. J Piao, "Uniqueness of Common Fixed Point for a Family of Mappings with $\phi$-Contractive Condition in 2Metric Spaces," Applied Mathematics, Vol. 3, 2012, pp. 73-77. doi:10.4236/am.2012.31012

[9] M. Abbas and G. Jungck, "Common Fixed Point Results for Noncommuting Mappings without Continuity in Cone Metric Spaces," Journal of Mathematical Analysis and Applications, Vol. 341, No. 1, 2008, pp. 416-420. doi:10.1016/j.jmaa.2007.09.070

[10] Y. Han and S. Y. Xu, "New Common Fixed Point Results for Four Maps on Cone Metric Spaces," Applied Mathematics, Vol. 2, 2011, pp. 1114-1118. doi:10.4236/am.2011.29153

[11] C. D. Bari and P. Vetro, “ $\phi$-Pairs and Common Fixed Points in Cone Metric Spaces," Rendiconti del Circolo Matematico Palermo, Vol. 57, 2008, pp. 279-285. 